\title{
Nivolumab Therapy for Synchronous ALK-Positive Lung Cancer and Gastric Cancer
}

\author{
Masahiro Yamasaki ${ }^{a} \quad$ Naomi Saito $^{a} \quad$ Yu Hada $^{b} \quad$ Sayaka Miyamoto $^{b}$ \\ Hideharu Okanobu ${ }^{b}$ Naoya Ikeda ${ }^{c}$ Wakako Daido ${ }^{a}$ Sayaka Ishiyama ${ }^{a}$ \\ Naoko Deguchi ${ }^{a} \quad$ Masaya Taniwaki $^{\mathrm{a}}$ Nobuyuki Ohashi, ${ }^{\mathrm{a}}$ \\ ${ }^{a}$ Department of Respiratory Disease, Hiroshima Red Cross Hospital \& Atomic Bomb \\ Survivors Hospital, Hiroshima, Japan; ${ }^{b}$ Department of Gastroenterology, Hiroshima Red \\ Cross Hospital \& Atomic Bomb Survivors Hospital, Hiroshima, Japan; 'Department of \\ Pulmonary Medicine and Clinical Immunology, Dokkyo Medical University School of \\ Medicine, Tochigi, Japan; ${ }^{\mathrm{d} O h a s h i}$ Clinic, Hiroshima, Japan
}

\section{Keywords}

Nivolumab · Immune checkpoint inhibitor - Driver oncogene $\cdot A L K \cdot$ Smoking $\cdot$ Multiple primary malignant tumors · Non-small cell lung cancer $\cdot$ Gastric cancer

\begin{abstract}
Nivolumab is an immune checkpoint inhibitor with demonstrated efficacy against several malignant tumors. Alterations in driver oncogenes such as EGFR and $A L K$ are a poor prognostic factor in nivolumab therapy for non-small cell lung cancer (NSCLC), whereas a smoking history is a well-known, favorable prognostic factor. However, an efficacy of nivolumab therapy for multiple primary malignant tumors (MPMTs) has not been reported, and its efficacy for driver oncogene-positive NSCLC in smokers is unclear. Herein, we report the case of a patient with a history of heavy smoking who developed synchronous ALK-positive NSCLC and gastric cancer that responded to nivolumab therapy. A 76-year-old man who was a heavy smoker presented to our hospital with symptoms of hoarseness and dysphagia. He was ultimately diagnosed with $A L K$-positive advanced NSCLC. An ALK inhibitor (alectinib) was administered, and the lung cancer lesions showed improvement. The alectinib therapy was continued for 5 months. Thereafter, the lesions in the left lower lobe of the lung showed
\end{abstract}


Yamasaki et al.: Nivolumab Therapy for Synchronous $A L K$-Positive Lung Cancer and Gastric Cancer

regrowth. During the same period, the patient experienced epigastric pain. Gastrointestinal endoscopy examination revealed gastric cancer. He was administered nivolumab to treat both the lung cancer and the gastric cancer. Two months later, both the lung lesions and the gastric lesions had diminished in size. Nivolumab therapy might be an effective therapy for synchronous MPMTs and NSCLC in heavy smokers, even if the lung cancer possesses driver oncogene mutations.

\section{Introduction}

Nivolumab, an anti-programmed cell death 1 (PD-1) antibody, is an immune checkpoint inhibitor with proven efficacy against several malignant tumors, including malignant melanoma [1], non-small cell lung cancer (NSCLC) [2, 3], renal cell carcinoma [4], classic Hodgkin lymphoma [5], gastric cancer [6], and urothelial carcinoma [7]. However, an efficacy of nivolumab therapy for multiple primary malignant tumors (MPMTs) has not been reported.

The prognostic factors of nivolumab therapy for NSCLC are uncertain. However, the results of subset analyses of clinical trial data revealed that driver oncogene alterations such as epidermal growth factor receptor (EGFR) mutation are poor prognostic factors [3]. Therefore, it is recommended that patients with EGFR and $A L K$ (anaplastic lymphoma kinase) aberrations should preferentially be treated with approved targeted therapy $[3,8]$. In addition, a smoking history is a well-known favorable prognostic factor in nivolumab therapy $[3,9]$.

Herein, we report the case of a heavy smoker with synchronous double primary $A L K$ positive NSCLC and gastric cancer that responded to nivolumab therapy.

\section{Case Presentation}

A 76-year-old man with a 159 pack-year smoking history presented to our hospital with the chief complaints of hoarseness, dysphagia, and weight loss. Otolaryngological examination revealed left vocal cord paralysis. He was examined using contrast-enhanced chest computed tomography (CT), which revealed multiple nodules in both lungs and lymphadenopathy of the bilateral hilar regions, mediastinum, and neck. After further examination, he was diagnosed with adenocarcinoma of the lung (cT1aN3M1a, stage IV) (Fig. 1a, b). The lung cancer was diagnosed as $A L K$ fusion gene positive using immunohistochemical staining and FISH (fluorescence in situ hybridization) at a commercial laboratory (SRL, Tokyo, Japan).

Oral alectinib, an $A L K$ inhibitor, was administered at $600 \mathrm{mg}$ daily. Two months later, a contrast-enhanced CT revealed improvement of the lung cancer lesions (Fig. 1c). The symptoms of hoarseness, dysphagia, and weight loss gradually improved. The alectinib therapy was continued for a total of 5 months.

Thereafter, the lesions in the left lower lobe of the lung showed regrowth (Fig. 1d). The patient developed symptoms of cough, hemoptysis, and epigastric pain. He underwent upper gastrointestinal endoscopy examination and was diagnosed with adenocarcinoma of the stomach (cT1bN0M0, stage IA) (Fig. 2a-c). The gastric cancer was diagnosed as ALK negative using immunohistochemical staining and FISH, and the histopathological findings of the gastric cancer were different from those of the lung cancer (Fig. 1b, Fig. 2c). This indicated that the gastric cancer was not a metastasis of the lung cancer. The gastric cancer was inoperable, because the lung cancer was advanced and uncontrolled. 
To treat both the lung cancer and the gastric cancer, the patient was administered nivolumab $3 \mathrm{mg} / \mathrm{kg}$ every 2 weeks. Five weeks later, the lung lesions showed marked improvement on CT examination (Fig. 3a). The symptoms of cough, hemoptysis, and stomach discomfort also improved. In addition, 2 months after the start of nivolumab administration, the gastric cancer was examined using upper gastrointestinal endoscopy, and the lesion had diminished in size (Fig. 3b, c). No adverse event related to the nivolumab therapy occurred. To date, the patient is alive and adverse event free and has continued nivolumab for a total of 3 months.

\section{Discussion}

In this study, we made 2 important clinical observations. First, nivolumab therapy might be effective for synchronous MPMTs. This is the first report describing the efficacy of nivolumab therapy for a patient with synchronous MPMTs. The main cancers that comprise MPMTs are gastrointestinal, lung, breast, urinary tract, and head and neck cancers [10]. These cancers mirror those that respond to nivolumab therapy. In the present case, the MPMTs were NSCLC and gastric cancer, both of which have been shown to respond to nivolumab therapy $[3,6]$. In the present case, nivolumab therapy was effective for both the lung cancer and the gastric cancer; thus, it might be a good alternative therapy for synchronous MPMTs.

Second, nivolumab therapy might be effective for NSCLC in heavy smokers, even if the lung cancer is $A L K$ fusion gene positive. Nivolumab was not more effective than docetaxel in patients with EGFR mutation-positive NSCLC [3]. However, it is estimated that the mutation burden is similarly high in driver oncogene-positive and driver oncogene-negative NSCLC in patients who are heavy smokers. The median progression-free survival (PFS) time of patients treated with alectinib was estimated to be longer than 29 months [11]. The PFS time in the present case was 5 months, which is relatively short. The reason for this short PFS time might have been the high mutation burden. It was reported that anti-PD-1 therapy was more effective for tumors with a higher mutation burden [12, 13]. Therefore, anti-PD-1 therapy might be effective for $A L K$-positive NSCLC in heavy smokers, especially for patients with a short PFS time treated with alectinib.

Because no specific chemotherapy regimen for MPMTs has been established, common chemotherapy regimens for each primary cancer are used empirically. It is believed that environmental factors are a cause of MPMTs [10]. The environmental factors include smoking, ultraviolet radiation, and ionized radiation, which induce various types of DNA damage and lead to carcinogenesis [14]. As described above, anti-PD-1 therapy is more effective against tumors with a high mutation burden. Therefore, anti-PD-1 therapy might be effective for MPMTs related to environmental factors, including smoking. To evaluate the efficacy of anti-PD-1 therapy for MPMTs, a prospective study is required.

\section{Conclusions}

We report the case of a patient with a history of heavy smoking who developed synchronous double primary $A L K$-positive NSCLC and gastric cancer that responded to nivolumab therapy. Nivolumab therapy might be a potential therapy for synchronous 
Yamasaki et al.: Nivolumab Therapy for Synchronous ALK-Positive Lung Cancer and Gastric Cancer

MPMTs and for NSCLC in heavy smokers, even if the lung cancer possesses driver oncogene mutations such as EGFR and ALK aberrations.

\section{Acknowledgements}

We would like to thank Editage (www.editage.jp) for English language editing.

\section{Statement of Ethics}

The authors have no ethical conflicts to disclose.

\section{Disclosure Statement}

The authors declare that they have no conflicts of interest.

\section{References}

1 Robert C, Long GV, Brady B, et al: Nivolumab in previously untreated melanoma without BRAF mutation. N Engl J Med 2015;372:320-330.

2 Brahmer J, Reckamp KL, Baas P, et al: Nivolumab versus docetaxel in advanced squamous-cell nonsmall-cell lung cancer. N Engl J Med 2015;373:123-135.

-3 Borghaei H, Paz-Ares L, Horn L, et al: Nivolumab versus docetaxel in advanced nonsquamous nonsmall-cell lung cancer. N Engl J Med 2015;373:1627-1639.

-4 Motzer RJ, Escudier B, McDermott DF, et al: Nivolumab versus everolimus in advanced renal-cell carcinoma. N Engl J Med 2015;373:1803-1813.

5 Younes A, Santoro A, Shipp M, et al: Nivolumab for classical Hodgkin's lymphoma after failure of both autologous stem-cell transplantation and brentuximab vedotin: a multicentre, multicohort, single-arm phase 2 trial. Lancet Oncol 2016;17:1283-1294.

-6 Kang YK, Satoh T, Ryu MH, et al: Nivolumab (ONO-4538/BMS-936558) as salvage treatment after second or later-line chemotherapy for advanced gastric or gastro-esophageal junction cancer (AGC): a double-blinded, randomized, phase III trial. J Clin Oncol 2017;35(suppl 4S):abstract 2.

-7 Sharma P, Retz M, Siefker-Radtke A, et al: Nivolumab in metastatic urothelial carcinoma after platinum therapy (CheckMate 275): a multicentre, single-arm, phase 2 trial. Lancet Oncol 2017;18:312-322.

-8 Kazandjian D, Suzman DL, Blumenthal G, et al: FDA approval summary: nivolumab for the treatment of metastatic non-small cell lung cancer with progression on or after platinum-based chemotherapy. Oncologist 2016;21:634-642.

-9 Sacher AG, Gandhi L: Biomarkers for the clinical use of PD-1/PD-L1 inhibitors in non-small-cell lung cancer: a review. JAMA Oncol 2016;2:1217-1222.

10 Xu LL, Gu KS: Clinical retrospective analysis of cases with multiple primary malignant neoplasms. Genet Mol Res 2014;13:9271-9284.

11 Takeuchi K, Togashi Y, Kamihara Y, et al: Prospective and clinical validation of ALK immunohistochemistry: results from the phase I/II study of alectinib for ALK-positive lung cancer (AF001JP study). Ann Oncol 2016;27:185-192.

12 Lawrence MS, Stojanov P, Polak P, et al: Mutational heterogeneity in cancer and the search for new cancer-associated genes. Nature 2013;499:214-218.

$\checkmark 13$ Rizvi NA, Hellmann MD, Snyder A, et al: Cancer immunology. Mutational landscape determines sensitivity to PD-1 blockade in non-small cell lung cancer. Science 2015;348:124-128.

14 Park JS, Kim HL, Kim YJ, et al: Human AP endonuclease 1: a potential marker for the prediction of environmental carcinogenesis risk. Oxid Med Cell Longev 2014;2014:730301. 


\section{Case Reports in Oncology}
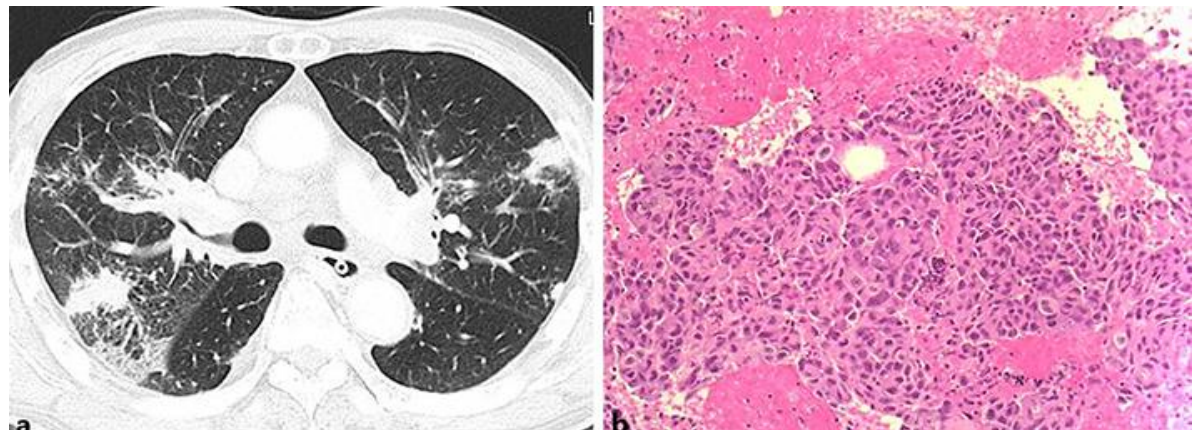

a. (h.

(C) 2017 The Author(s). Published by S. Karger AG, Basel www.karger.com/cro

Yamasaki et al.: Nivolumab Therapy for Synchronous ALK-Positive Lung Cancer and Gastric Cancer
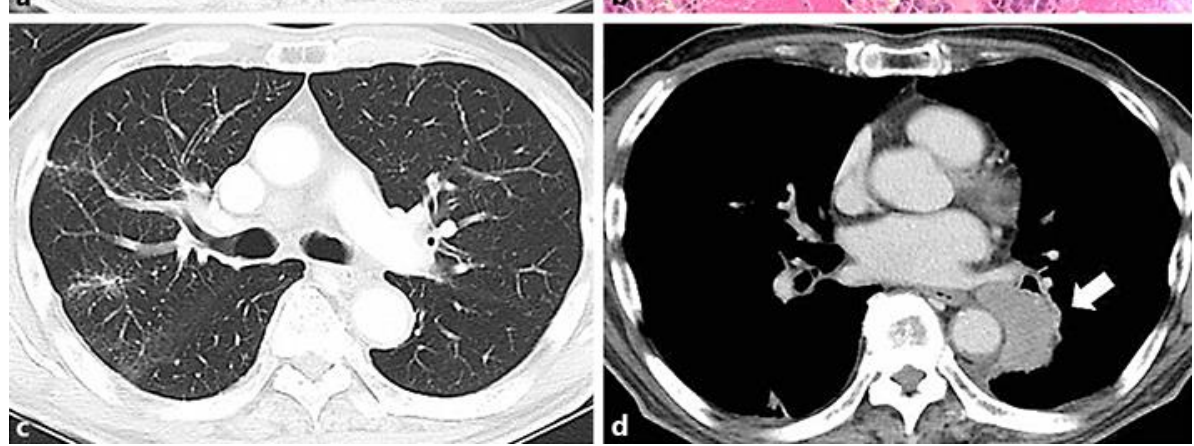

Fig. 1. Initial chest computed tomography (CT) and microscopic findings of lung cancer, and the chest CT findings after 2 and 5 months of alectinib. a Multiple masses and nodules are seen in both lungs. The lesions were diagnosed as $A L K$-positive lung adenocarcinoma. $\mathbf{b}$ The lung cancer specimen shows an alveolar or sheet-like structure. c After 2 months of alectinib administration, all lesions are markedly improved. d After 5 months of alectinib administration, the lesions in the left lower lobe of the lung show regrowth (arrow). 


\section{Case Reports in Oncology}
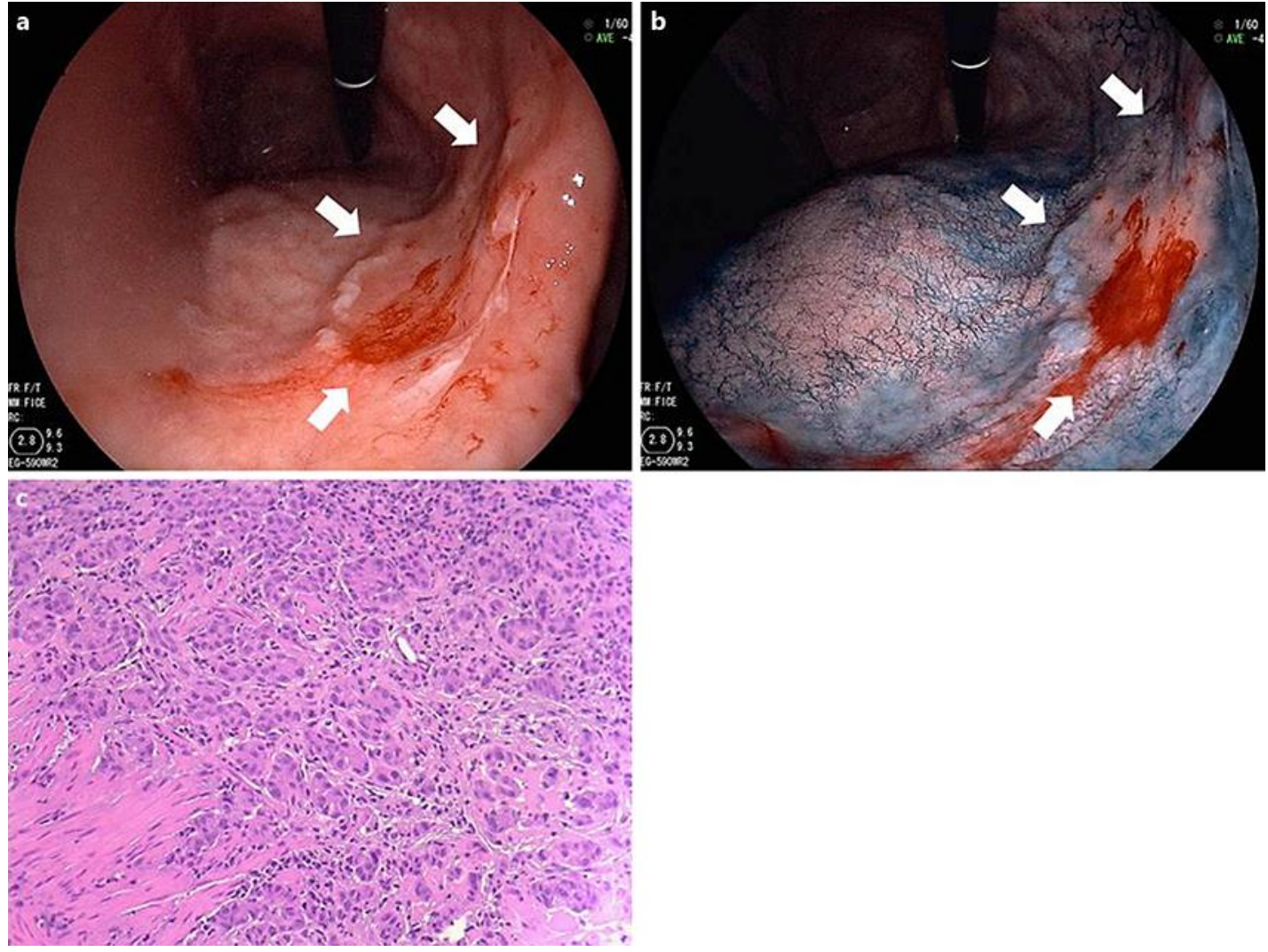

www.karger.com/cro

Yamasaki et al.: Nivolumab Therapy for Synchronous $A L K$-Positive Lung Cancer and Gastric Cancer

Fig. 2. Endoscopic and microscopic findings of gastric cancer. a Conventional endoscopic finding (arrows). b Dye endoscopic finding by the indigo carmine contrast method. Prior to nivolumab administration, endoscopy revealed a depressed lesion in the gastric angle region, which was diagnosed as gastric adenocarcinoma (arrows). c Microscopically, the gastric cancer specimen has an alveolar or acinus-like structure. The histopathological findings of the gastric cancer are different from those of the lung cancer. 


\section{Case Reports in Oncology}
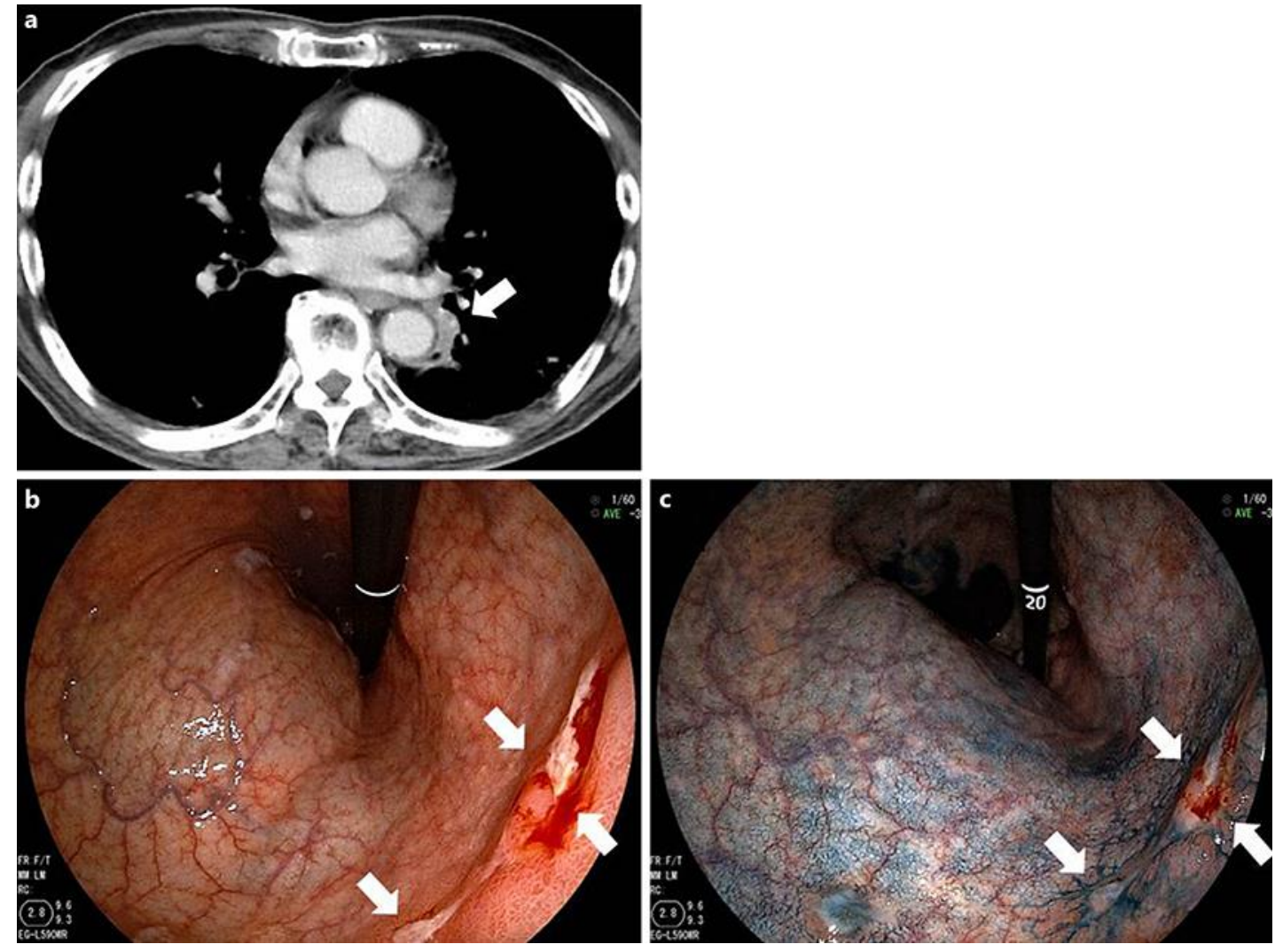

Fig. 3. CT findings of lung cancer as well as endoscopy of gastric cancer after nivolumab administration. a After 5 weeks of nivolumab administration, the lung lesions show marked improvement (arrow). b Conventional endoscopic finding (arrows). c Dye endoscopic finding by the indigo carmine contrast method. After 2 months of nivolumab administration, the gastric lesion diminished in size (arrows). 